\title{
Practical Approaches for Size Estimation of High-Risk Groups in HIV/AIDS Control Program in Iran
}

\author{
Salman KHAZAEI ${ }^{1}$, Ensiyeh JENABI ${ }^{2}$, *Shahab REZAEIAN ${ }^{3}$ \\ 1. Research Center for Health Sciences, Hamadan University of Medical Sciences, Hamadan, Iran \\ 2. Pediatric Developmental Disorders Research Center, Hamadan University of Medical Sciences, Hamadan, Iran \\ 3. Research Center for Environmental Determinants of Health, Kermanshah University of Medical Sciences, Kermanshah, Iran \\ *Corresponding Author: Email: shahab.rezayan@gmail.com
}

(Received 11 Jan 2018; accepted 20 Jan 2018)

\section{Dear Editor-in-Chief}

Acquired Immune Deficiency Syndrome (AIDS) is an emerging disease which has become known as the plague of the century. The estimated number of infected people with HIV in Iran is 71000 (53000-100000) in 2012 (1). The prevalence in the overall population is below $1 \%$; this rate, however, has surpassed $5 \%$ in some high-risk groups such as injecting drug users (IDUs) (2). The country's HIV epidemic is concentrated among IDUs, $68.1 \%$ of cases are due to needle-sharing versus $12.7 \%$ to sexual transmission. The highest trend of HIV was estimated to be a shift from IDUs to female sex workers (FSWs), and newly infected cases via unsafe sex will increase in the future (1).

Estimating the population size of hard to reach subgroups is an important entity in public health, especially in the HIV/AIDS control program (3). Having reliable estimates of the size of these groups (especially FSWs and IDUs in our country) is necessary for better tracking the epidemic, program planning, advocacy and enough resource allocations (3). This information is more need for countries like Iran with a concentrated HIV epidemic among IDUs and with limited resources (2).

There are few published statistics is available about population size of these groups using a standard and validated methodology in Iran (4-6). In order to improve the current surveillance system, it is crucial to having acceptable population size of these groups. However, such groups are hidden in the community, mainly because of strong stigmatization and legal punishment. Therefore they have reluctant to be identified as such and the number of them is prone to underestimation (7).

Special techniques have been introduced for size estimation of these groups including capturerecapture (CRC), multiplier (MP), and Networks Scale Up (NSU) techniques (8). Direct CRC and MP methods need direct contact between research team and high-risk groups. These techniques are appropriate to estimate sizes in a close population. However, for planning, we need to have national estimates. Indirect CRC and NSU which needs no direct contact with individuals with high-risk groups (8).

Some challenges like stigma and discrimination against such population in Iran with an Islamic culture have caused the direct estimation techniques to approximate their population size is inappropriate, also because of their small population size in different societies, any survey for direct estimation requires a very large sample size, which is also not often feasible. Although the indirect size estimation methods are commonly used in our country for these groups (9), the underestimate rate of the population still remains considerable in Iran. 


\section{Conflict of interest}

The authors declare that there is no conflict of interests.

\section{References}

1. Islamic Republic of Iran: AIDS progress report on monitoring of the United Nations General Assembly Special Session on HIV and AIDS. Tehran: National AIDS Committee Secretariat, Ministry of Health and Medical Education;2012. http://www.unaids.org/sites/default/files /country/documents/IRN_narrative_repo rt_2015.pdf

2. Shokoohi M, Baneshi MR, Haghdoost AA (2010). Estimation of the active network size of Kermanian Males. Addict Health, 2(3-4):81-8.

3. Karami M, Khazaei S, Poorolajal J et al (2017). Estimating the Population Size of Female Sex Worker Population in Tehran, Iran: Application of Direct Capture-Recapture Method. AIDS and Behav, 21(8):2394-400.

4. Khazaei S, Poorolajal J, Mahjub H et al (2012). Estimation of the frequency of intravenous drug users in Hamadan City, Iran, using the capture-recapture method. Epidemiol Health,34:e2012006.

5. Karami M, Mirzaei M, Khazaei S et al (2017). Estimating the Population of Female Sex Workers in Hamadan, Western Iran, 2014. Int J High Risk Behav Addict, 6(4): e63195

6. Shokoohi M, Baneshi MR, Haghdoost AA (2012). Size estimation of groups at high risk of HIV/AIDS using network scale up in Kerman, Iran. Int J Prev Med, 3(7):471-6.

7. Mathers BM, Degenhardt L, Phillips B et al (2008). Global epidemiology of injecting drug use and HIV among people who inject drugs: a systematic review. Lancet, 372(9651):1733-45.

8. Vadivoo S, Gupte MD, Adhikary R et al (2008). Appropriateness and execution challenges of three formal size estimation methods for high-risk populations in India. AIDS, 22 Suppl 5:S137-48.

9. Maghsoudi A, Baneshi MR, Neydavoodi M, Haghdoost A (2014). Network scale-up correction factors for population size estimation of people who inject drugs and female sex workers in Iran. PLoS One, 9(11):e110917. 